\title{
EDITORIAL
}

\section{Intracoronary stenting: developments since the NICE report}

A H Gershlick

Heart 2002;87:187-190

Stenting has become standard treatment for patients undergoing percutaneous coronary intervention. Clinical impressions continue to support this as a user friendly, safe, and efficacious technology

$\mathrm{T}$ he National Institute for Clinical Excellence (NICE) was established in the light of a perceived need for a regulatory body to review the efficacy of existing and new treatments and to appraise technological developments. It was seen as being important for the following reasons: (1) there was and is slow uptake, even of innovations of perceived benefit; (2) judgements on the interpretation or significance of the evidence can be different in different parts of the country, resulting in variations in the access for patients to the new treatments with the widespread perception of inequity; (3) wasteful use of resources can occur when treatments are used outside the range in which they are clinically cost effective, at the expense of alternative uses of those resources which could give greater benefits to patients.

The key functions of NICE are technology appraisal of both new and existing interventions, in order to assess clinical benefit and cost effectiveness. Guidelines are issued and information disseminated. It is expected that progress will be audited. Advocates suggest that the good things about NICE include the fact that it was badly needed, and that it is transparent, has authority, and it has been able to rationalise the often difficult debate between clinicians and purchasers. It produces evidence based reports. Its detractors would claim it takes evidence from experts who had "something to sell", and that it tends to depend too heavily on randomised clinical trials, which select populations, rather than to also use registry data. Recommendations come with no money attached.

Overall, however, the establishment and work of NICE has been generally welcomed. For better or worse NICE is seen in Europe as a lead model for health and technology assessment (HTA) groups, as compared to some parts of Europe where such groups are seen as an academic exercise or produce reports that sit on shelves. HTA groups such as NICE and ANAES (Agence Nationale d'Accreditation et d'Evaluation en Sante) in France are seen as being much more influential since they impact on clinical practice and reimbursement.

In 1999 NICE examined the use of coronary stents as the first in a number of cardiovascular exercises, being followed by glycoprotein IIb/IIIa receptor blockers, and implantable defibrillators.
The reasons for examining coronary stents were the substantial increase in their use (percutaneous coronary intervention (PCI) procedures: 9933 in 1991, stent rate $2.7 \% ; 28133$ in 1999, stent rate $79 \%$ ) and consequently their impact on budgets, concern among some clinicians and purchasers about clinical benefits and cost effectiveness, and because heart disease had been a declared government priority. The NICE report published in May 2000 came out very positively in favour of stenting. It recommended in essence that in patients undergoing PCI stents should be used routinely, that their availability should encourage the use of PCI as opposed to coronary artery bypass grafting, but that the evidence for their use in vessels $<2.5 \mathrm{~mm}$ was not adequate at that time. Use of stents in vein grafts was considered an acceptable treatment.

Stenting was not at the time of the report a clinical innovation, having been increasingly used before the NICE review. Many physicians felt the report only reflected and gave approval to a procedure that was in their minds an important evidence based adjunct to balloon angioplasty: it reflected what they believed to be an improvement in good clinical practice at the individual

Abbreviations: ADMIRAL, abciximab before direct angioplasty and stenting in myocardial infarction regarding acute and long-term follow-up; AMIST, angioplasty versus minimally invasive surgery trial; ANAES, Agence Nationale d'Accreditation et d'Evaluation en Sante; ARTS, arterial revascularization therapy study; BESMART, Bestent in small arteries; CABG, coronary artery bypass graft; CADILLAC, controlled abciximab and device investigation to lower late angioplasty complications; ELUTES, evaluation of taxol eluting stent trial; EPISTENT, evaluation of platelet GP Ilb/llla inhibitor for stenting; ESPRIT, European study of prevention of reocclusion after initial thrombolysis; HTA, health and technology assessment; INHIBIT, inhibit restenosis intervention with $\beta$ radiation trial; ISAR-SMART,

intracoronary stenting or angioplasty for restenosis reduction in small arteries; LMS, left main stem; LVEF, left ventricular ejection fraction; MACE, major adverse cardiac event; MI, myocardial infarction; NICE, National Institute for Clinical Excellence; RAP, restenosis en arterias pequenas; RAVEL, randomized study with sirolimus coated $B X$ velocity balloon expandable stent in the treatment of patients with de novo native coronary lesions; RCT, randomised controlled trial; SAFER, saphenous vein graft angioplasty free of emboli randomized trial; SISA, stents in small arteries; SIRUS, sirolimus; SLIDE, selected lesion indication for direct stenting; SOS, stent or surgery; START, stents and radiation therapy trial; TARGET, do tirofiban and Reopro give similar efficacy outcomes trial; TIMI, thrombolysis in myocardial infarction; TLR, target lesion revascularisation; TVR, target vascularisation rate; ULTIMA, unprotected left main trunk intervention multi-center assessment; VBT, vascular brachytherapy 
patient level. In most centres in 2001 stents are used in > 70\% of interventional procedures. Those who continue to struggle with purchasers are presumably using the NICE guidelines to ensure they get the best appropriate care for their PCI patients. Some clinicians remain sceptical ${ }^{1}$ but the data on which they base such scepticism could in itself be criticised as being flawed and based on old technology in selected patients.

Despite the important contribution stenting has made, there are still areas of debate and data for the expanded role of stents are continually being sought with new trials being undertaken.

\section{STENTING AND AREAS OF CONTENTION Small vessel disease}

Previous observational studies have suggested high restenosis rates (up to $45 \%$ ) in those patients with small vessel disease. Five recent studies have produced conflicting results. Park and colleagues $^{2}$ assigned 60 patients with reference vessel size $<3 \mathrm{~mm}$ to balloon and 60 to stents. The six month angiographic restenosis rate was $30.9 \%$ in the balloon group of patients and $35.7 \%$ in the stent group. The target lesion revascularisation rate was low in both groups $(5 \%$ balloon and 3\% stent). The SISA $\operatorname{trial}^{3}(\mathrm{n}=325)$ showed a trend only towards less adverse outcomes with stent versus balloon in vessels $<2.9 \mathrm{~mm}$. This was because the acute gain with stenting ( $1.37 \mathrm{~mm} v 0.91 \mathrm{~mm}, \mathrm{p}=0.0001)$ was offset by the greater late loss in the stent group $(28.5 \% v 18.4 \%, \mathrm{p}=0.0002)$. In the setting of small vessels such late loss caused by intimal tissue cannot be accommodated so easily.

The BESMART study ( $\mathrm{n}=381$ ) on the other hand (using the same "Bestent" stent) demonstrated a restenosis rate of $22.7 \%$ in the stent group versus $48.8 \%$ in the balloon group $(\mathrm{p}<0.0001)$ and a target lesion revascularisation (TLR) rate of $13 \%$ versus $25 \%(p<0.016),{ }^{4}$ whereas ISAR-SMART showed no benefit from small vessel stenting (35.7\% v 37.4\%, vessel size $2.0-2.8 \mathrm{~mm}$ ). ${ }^{5}$ The RAP study (vessel size $2.2-$ $2.7 \mathrm{~mm}$ ) demonstrated a restenosis rate of $27 \%$ in the stent group versus $37 \%$ in the balloon group $(p=0.04) .{ }^{6}$ Thus two trials show benefit and three show no benefit compared to balloon. While randomised controlled trials (RCTs) are important, there are a number of registries that demonstrate low clinical event rates with stents in small vessels. Thus in the Biocompatibles small vessel registry the major adverse cardiac event (MACE) rate was only 3.55 at one month and the TLR rate only $0.7 \%$. The mean reference diameter of this European registry was $2.2 \mathrm{~mm}$.

The value of stenting small vessels is clearly unresolved. Why there should be such differences between the RCT trials is unclear. Certainly there were differences in reference vessel diameter between "positive" studies and "negative" studies, although counter-intuitively they were smaller in the positive studies. The focal nature of the lesion in the positive studies could also imply that stenting should be reserved for such lesions. Specific stent design in terms of strut dimensions and cell size may also be important factors.

It is clear that physicians may not wish to exclude patients from the potential benefit of stenting based on vessel size alone. It is likely therefore that it is in this group that newer innovations, such as new stent designs or drug eluting stents, may have most impact since intimal hyperplasia will always have greater impact on the smaller lumen.

Currently clinicians tend to stent vessels with reference sizes between $2.5-3 \mathrm{~mm}$, providing there are few other high risk features for restenosis (such as diffuse disease). At this time this group of patients need to be the target for any innovative strategies, which should be studied in well designed RCTs.

\section{Left main stem stenting}

It had always been regarded as taboo to undertake PCI on unprotected left main stem (LMS) disease, and NICE implied that surgery was the desired option. However, a number of groups world wide are, through the use of registries, identifying the real overall risks of PCI in such patients and in whom intervention could be deemed acceptable. Early studies such as that by Park suggested excellent results in those who could be regarded as low risk ( $100 \%$ success rate, $17 \%$ clinical recurrence at six months, and only one death), ${ }^{7}$ and Barragan reported three deaths out of 15 patients considered high risk, but 0 out of 17 in the low risk group.

Ellis has reported on the ULTIMA registry on 279 consecutive patients who had LMS PCI at 25 centres between 1993 and 1998. The outcome appeared to be dependent on patient characteristics. Forty six per cent of these patients were deemed inoperable or at high surgical risk. Overall (13.7\%) died in-hospital, and the rest were followed for a mean of 19 months. The one year all cause mortality was $24.2 \%$, with a cardiac mortality rate of $20.2 \%$ and an acute myocardial infarction (MI) rate of $9.8 \%$, and $9.4 \%$ need for coronary artery bypass graft surgery (CABG). Independent correlates of all cause mortality were: left ventricular ejection fraction (LVEF) $\leqslant 30 \%$, mitral regurgitation grade 3 or 4 , presentation with MI and shock, creatinine $\geqslant 2.0 \mathrm{mg} / \mathrm{dl}$, and severe lesion calcification. In the $32 \%$ of patients $<65$ years old, with LVEF $>30 \%$ and without shock, there were no periprocedural deaths, and the one year mortality was only $3.4 \%$.

Who should undergo unprotected LMS PCI? Clearly those who are rejected surgically and who are severely disabled by angina could be considered after full and frank discussion with the patient and their family. In young patients with ideal anatomy LMS PCI is also being undertaken. The general rules would appear to be: ostial or disease in short LMS is high risk, especially if calcified, as are those patients with further multivessel disease, or reduced ejection fraction. Anything other than a perfect PCI result (including the use of intravascular ultrasound) is unacceptable.

Trials are difficult to do and registries are ongoing.

\section{Bifurcation lesions}

The best treatment for bifurcation disease is unresolved; since the NICE report fewer interventional procedures for this condition are being undertaken world wide. Some interventionalists question whether PCI is the treatment of choice, because of technical issues and the high incidence of acute and chronic events. Stent deployment in both arms of the bifurcation, or the stenting of one and ballooning of the other depending on the presence of disease or the result of intervention, are current topics for debate. While some authors have reported very high restenosis rates, Lefevre ${ }^{10}$ reported MACE rates of between $17.1-29.2 \%$ which was in part influenced by the experience of the operator. The use of so-called "kissing" balloons appeared to influence the outcome beneficially. Others have shown that stenting of the side branch may not be essential, but choosing which to stent and which to leave is the subject of several proposed studies. Bifurcation stenting is currently less enthusiastically undertaken.

\section{Direct stenting}

Direct stenting may have significant advantages over routine procedure which uses balloon predilatation first. There appears little doubt that tissue responses to the vessel wall may be influenced by damage induced by the balloon inflation (and stent deployment). If this trauma could be reduced, less tissue response and restenosis should occur. Additionally the procedure should be cheaper requiring a balloon-stent unit only. There have been a number of reports outlining the feasibility of such a strategy. Hamon recently published the outcome of 122 "carefully selected" patients. Factors such as calcification and tortuosity need to be taken into account when direct stenting is considered. Procedural success was $96 \%{ }^{11}$ In five cases it was not possible to deliver the stent 
through the undilated lesion and the stent was retrieved, but in two cases the stent was lost in the peripheral circulation. The authors rightly reported the need for a controlled trial and one UK study (the SLIDE trial) is to be published soon. Unpublished data from this study indicate that attainment of a $<30 \%$ residual diameter stenosis was achieved in $94 \%$ of direct stented patients, with no difference in six month MACE between direct and predilatation groups (7.1\% and 6.7\%). The direct stent strategy resulted in significantly less balloon use $(\mathrm{p}<0.001)$ and in $80 \%$ the stent delivery system was the sole treatment strategy ( $\mathrm{R}$ Stables, personal communication). In general, this and other studies suggest that if the operator feels able to select those lesions suitable for direct stent deployment and does so then this is a safe and cost saving exercise.

\section{STENTS VERSUS SURGERY}

There have been a number of randomised studies comparing stenting with surgery for multivessel disease (ARTS, SOS). The one year results of the ARTS trial $(n=1200)$ which compared stenting (mean (SD) 2.7 (0.2) stents per patient) to surgery (2.8 (1.1) anastomoses per patient) in multivessel disease have been published. ${ }^{12}$

The event-free survival was higher in the surgical patients $(87.3 \% \vee 73.3 \%)$, entirely because of the need for reintervention in the stented patients.

The UK based SOS trial has been completed and the results clearly show that even in the era of stenting early outcome after coronary surgery is better than after PCI when need for revascularisation is the end point ( $17 \%$ PCI $v 4 \%$ surgery at one year, $\mathrm{p}<0.001)$.

Comparisons between angioplasty plus stenting and minimally invasive surgery to the left anterior descending artery are underway (the AMIST study). Recruitment is slow for a number of logistical reasons and to date 88 patients have been randomised.

\section{CHALLENGES FOR PCI}

It has become clear that the most important barrier to overcome is the elimination of in-stent restenosis (ISR), which leads to the need for repeat intervention. Additional goals include finding the right place for PCI in the treatment of acute myocardial infarction and making the procedure safer in higher risk cases, such as vein graft stenting.

\section{Dealing with in-stent restenosis}

ISR remains one of the important remaining challenges for investigators. Treatment in the form of vascular brachytherapy (VBT) is available. ISR rates are reduced by about $60 \%$ and the clinical outcome (target revascularisation rate) by about $50 \%$ irrespective of whether this is delivered as a $\beta$ emitter or a $\gamma$ emitter. Two recently published trials $\left(\right.$ START $^{13}$ and INHIBIT ${ }^{14}$ ) specifically demonstrated that, compared to placebo, the use of ${ }^{90} \mathrm{Sr} / \mathrm{Y}$ and ${ }^{32} \mathrm{P}$ radionucleotide post-reballooning to deliver $16 \mathrm{~Gy}$ at $2 \mathrm{~mm}$ inside the vessel wall reduced the in-stent recurrence rate from $42.2 \%$ to $14.2 \%(\mathrm{p}<0.001)$ and $48 \%$ to $16 \%(\mathrm{p}<0.0001)$, respectively. VBT has less benefit when used as a primary treatment in non-stented vessels.

The main problem, however, is that VBT is secondary treatment-patients have to have developed ISR first. An alternative approach would be primary prevention.

There is no doubt that it is the aim of all clinical investigators to eliminate or significantly reduce the incidence of ISR, if only to reduce further the gap between PCI and surgery (currently $\sim 14 \%$, ARTS). The concept of drug eluting stents to reduce ISR is generating great interest. A number of agents in combination with different stents are currently undergoing clinical trials. Paclitaxel has specific properties against microtubules, promoting polymerisation of tubulin. It inhibits the disassembly of microtubules, which thus become very stable and dysfunctional, so inhibiting cell division. A European pilot safety study (ELUTES) was recently reported at the 2001 meeting of the American Heart Association, and showed 3\% binary restenosis in the treated arm versus $21 \%$ in controls.

A European based trial of sirolimus coated on Cordis Bstent (RAVEL study) has been conducted. Sirolimus is a naturally occurring macrocyclic lactone. It has been used as an immunosupressive agent in renal and islet grafting and for bone marrow transplantation. It binds to a specific cytosolic protein (immunophilin) found in target cells. This complex then binds to a specific regulatory kinase called the "mammalian target of rapamycin" (mTOR), inhibiting its activation, which in turn through inhibition of cell cycle progression suppresses cytokine stimulated T cell proliferation. However it has other effects, including inhibition of translation of cdk4/cyclin d and cdk2/cyclin E complexes, that are perhaps of greater potential for inhibiting in-stent restenosis. Early results are exciting, with the first human study reported to show no intimal hyperplasia at 12 months in 30 patients, ${ }^{15}$ and the RAVEL trial reporting $0 \%$ binary restenosis in treated patients versus $26 \%$ in controls (European Society of Cardiology 2001). The USA based SIRUS trial is half completed (550/ 1100 ) and the E-SIRUS study (longer, more complex lesions) is due to start mid 2001. Drug eluting stents will remain the focus of interest over the next 2-3 years.

Other stents with the same or other drugs are either in the early stages of clinical development or have started to be investigated in trials.

\section{Following previous coronary artery bypass grafting}

One further challenge is in the treatment of old grafts, where PCI can result in atherosclerotic debris occluding the distal vessel, producing the no-reflow phenomenon. A recent trial, the SAFER study, suggests that the outcome (in terms of enzyme defined MI) is better if a distal protection device is used. In this study 800 patients were randomised to either the Percusurge protection device or no protection device. The results were striking. The incidence of primary end point (MACE combined clinical end point defined as death, Q wave or non-Q wave MI, emergent bypass surgery, or repeat target vessel revascularisation) in the Percusurge group were 50\% less than in the control group during in-patient stay and at 30 days (cumulative MACE to 30 days: protection device $9.9 \%$, control $19.8 \%, p=0.001)$. The end point was driven by lower incidence non-Q wave infarcts; perhaps not surprisingly the incidence of enzyme increases $>3 \times$ normal was also significantly reduced .

\section{ACUTE CORONARY SYNDROMES, PCI, AND THE GLYCOPROTEIN IIb/IIIa RECEPTOR BLOCKERS}

The introduction of the glycoprotein (GP) IIb/IIIa inhibitors has had an impact on PCI and, in particular, stenting. In those patients undergoing PCI the situation at first appears clear. PCI disrupts plaques and the incidence of events are reduced with GP IIb/IIIa inhibitors. Whether the enzyme release, which was what drove the end points in the trials, has true longer term clinical significance ("enzyme bumps" or "prognostically important micro-infarcts") has been much debated. Consensus generally supports the belief that such enzyme release is probably bad and thus the use of GP IIb/IIIa inhibitors is recommended. The situation is confused, however, by the fact that while all three GP IIb/IIIa inhibitors (abciximab, eptifibatide, and tirofiban) have shown benefit compared to placebo, the TARGET trial ${ }^{16}$ appeared to demonstrate superiority of abciximab over tirofiban (event rate $5.7 \% v 7.2 \%$, respectively), while no comparison is available between eptifibatide and the other agents. Furthermore, a significant number of clinicians continue to be unconvinced of the value of such agents in low risk PCI (despite the NICE recommendations), the incidence of enzyme rise being in some clinicians' opinion 
less frequent and of less significance. Careful subgroup review of all the trials indicates that only one (EPISTENT) demonstrated benefit in routine cases and even then there may have been case selection, with those who would normally receive GP IIb/IIIa inhibitors not being randomised. The ESPRIT trial, ${ }^{17}$ which was designed as a planned PCI study, in fact showed benefit in the "unstable angina" group only (stable angina, 25\% reduction $7.2 \% \vee 5.4 \%, p=0.29$; acute coronary syndrome $>2$ days $48 \%$ reduction, $11.1 \%$ to $5.7 \%$, $\mathrm{p}=0.013$ ). The British Cardiac Intervention Society (BCIS) is currently planning a study to assess the value of these agents in routine stenting. Should a patient at high risk of acute coronary syndrome (ECG changes + troponin positive) be initiated on a GP IIb/IIIa inhibitor, the general consensus is that transfer and intervention during the infusion is better. If the patient cannot be transferred during that time then a decision will be taken about reinitiation of infusion, probably with abciximab.

There appears no doubt that PCI in acute MI trial patients improves outcome compared to thrombolysis ${ }^{18}$ and that stenting is better than balloon angioplasty alone. Two recent studies have addressed the value of adding a GP IIb/IIIa receptor blocker (abciximab). In the CADILLAC study ${ }^{19}$ patients were randomised to stenting plus abciximab, stenting, balloon plus abciximab, or balloon. The event rate (primary end point death, re-MI, disabling stroke or ischaemia driven TVR at six months) was reached in $10.8 \%, 10.9 \%, 20 \%$, and $20 \%$, respectively, suggesting that stenting is superior to balloon but that abciximab did not improve clinical outcome. This was different to the findings in the ADMIRAL study ${ }^{20}$ where the 30 day composite end point was achieved in $14.6 \%$ of 151 patients treated with stent plus placebo compared to $6.0 \%(\mathrm{p}=0.01)$ of 149 patients treated with stent plus abciximab. The difference in outcomes related to the better TIMI grade 3 flow in the treated group before $(16.8 \% \vee 5.4 \%, \mathrm{p}=0.01)$, immediately following $(95.1 \% v 86.7 \%, \mathrm{p}=0.04)$, and at six months $(94.3 \%$ $v 82.2 \%, \mathrm{p}=0.04)$ after the procedure.

\section{NICE RECOMMENDATIONS REVISITED}

Stenting has become standard treatment for patients undergoing PCI. Clinical impressions continue to support this as a user friendly, safe, and efficacious technology. Few patients need urgent surgery and many less patients come back to outpatients with a recurrence of symptoms now that stenting has become accepted treatment. Training time of juniors has been shortened. Clearly issues remain and trials continue to explore the full potential of stenting. Such data will be available to NICE. Ensuring appropriate application of intervention requires more than recommendations from an HTA, however; appropriate training, case selection, peer review, and perhaps a risk scoring system for appropriate referral need to be carefully considered.

\section{REFERENCES}

1 Karsch K R, Newby A C. Stent magic! The genie has escaped from the bottle. Heart 2000;84:469-70.

2 Park SW, Lee MK, Hong JJ, et al. Randomized comparison of coronary stenting with optimal balloon angioplasty for treatment of lesions in small coronary arteries. Eur Heart J 2000;21:1785-9.

3 Schalii M, Doucet S, Hilton D, et al. The SISA study: a randomised comparison of balloon angioplasty and stent to prevent restenosis in small arteries: 6 month angiographic and 12 month clinical outcome [abstract]. Circulation 2000;18:II-663.

4 Koning R, Khalife K, Commeau P, et al. The BESMART (Bestent in small arteries): in-hospital clinical and angiographic results [abstract]. Circulation 1999;100:I-503

5 Kastrati A, Schoming A, Dirschinger J, et al. A randomised trial comparing stenting with balloon angioplasty in small vessels in patients with coronary artery disease. Circulation 2000;102:2593-8.

6 Garcia E, Gomez-Recio M, Moreno R, et al. Stent reduces restenosis in small vessels. Results of the RAP study J Am Coll Cardiol 2001;37/suppl II):32-4.

7 Park SJ, Park SW, Hong MK, et al. Stenting of unprotected left main coronary artery stenoses: immediate and late outcome. J Am Coll Cardiol 1998;31:37-42.

8 Barragan P, Silvertri M, Simeoni JB, et al. Stenting in unprotected left main coronary artery: immediate and follow-up results [abstract]. Circulation 1996;94(suppl I):-1-672

9 Ellis SG, Tamai $\mathrm{H}$, Nobuyoshi $M$, et al. Contemporary percutaneous treatment of unprotected left main coronary stenoses. Initial results from a multicenter registry analysis 1994-1996. Circulation 1997;96:3867-72.

10 Lefevre T, Louvard Y, Morice MC, et al. Stenting of bifurcation lesions: classification, treatments, and results. Cath Cardiovasc Intervent 2000;49;274-83

11 Hamon $M$, Richardeau $Y$, Lecluse E, et al. Direct coronary stenting without balloon predilation in acute coronary syndromes. Am Heart J 1999:138:55-8.

12 Serruys PW, Unger F, Sousa JE, et al. Comparison of coronary artery bypass surgery and stenting for the treatment of multivessel disease. $N$ Engl J Med 2001;344:1117-24

13 Popma J, Heuser R, Suntharalingam M, et al for the START Investigators. Late clinical and angiographic outcomes after use of ${ }^{90} \mathrm{Sr} /{ }^{90} \mathrm{Y}$ beta radiation for the treatment of in-stent restenosis. Results from the stents and radiation therapy (START) trial [abstract]. J Am Coll Cardiol 2000;35:(suppl).

14 Waksman $\mathbf{R}$, Raizner A, Lansky A, et al. Beta radiation to inhibit recurrence of in-stent restenosis: study design, device and dosimetry details of the multicenter randomised double blind study [abstract]. Circulation 2000;102 (suppl II):II-667.

15 Sousa JE, Costa MA, Abizaid A, et al. Lack of neointimal proliferation after implantation of sirolimus-coated stents in human coronary arteries a quantitative coronary angiography and three-dimensional intravascular ultrasound study. Circulation 2000;102:54-7.

16 Topol EJ, Moliterno DJ, Herrmann HC, et al. Comparison of two platelet glycoprotein Ilb/llla inhibitors, tirofiban and abciximab, for the prevention of ischaemic events with percutaneous coronary prevention of ischaemic events with percutaneous coronar

17 The ESPRIT Investigators. Novel dosing regimen of epitifiatide in planned coronary stent implantation (ESPRIT): a randomised, placebo-controlled trial. Lancet 2000;356:2037-44.

18 Weaver WD, Simes R, Betriu A, et al. Comparison of primary coronary angioplasty and intravenous thrombolytic therapy for acute myocardial infarction: a quantitative review. JAMA 1997;278:2093-8.

19 Stone, et al. Stenting or balloon angioplasty with or without abciximab in acute MI. The CADILLAC study. Presented at American Heart Association 2000

20 Montalescot G, Barragan P, Wittenberg O, et al. Platelet glycoprotein llb/llla inhibition with coronary stenting for acute myocardial infarction. N Engl J Med 2001;344:1895-903. 


\title{
EDITORIAL
}

\section{Mechanical reperfusion therapy for acute myocardial infarction: Stent PAMI, ADMIRAL, CADILLAC and beyond}

\author{
B R Brodie, T D Stuckey
}

Heart 2002;87:191-192

\begin{abstract}
Mechanical reperfusion has gained increasing acceptance as a reperfusion strategy for acute myocardial infarction
\end{abstract}

M echanical reperfusion has gained increasing acceptance as a reperfusion strategy for acute myocardial infarction (AMI) as a result of data from numerous randomised trials showing superior outcomes. Recently, the Stent PAMI, ADMIRAL, and CADILLAC trials have documented further improvement in outcomes with the addition of adjunctive treatment with coronary stents and platelet glycoprotein IIb/IIIa inhibitors. ${ }^{1-3}$ Grines and colleagues, in the Stent PAMI trial, found that stents reduced target vessel revascularisation (TVR) and major adverse cardiac events (MACE) at six months compared to balloon angioplasty alone. ${ }^{1}$ (The Stent PAMI trial initially raised some concerns because the incidence of TIMI 3 flow was slightly lower following stenting versus balloon angioplasty, and there was a disturbing trend toward higher mortality in stented patients at six months. These concerns were resolved in the CADILLAC trial). Montalescot and colleagues, in the ADMIRAL trial, found that the use of the platelet glycoprotein IIb/IIIa inhibitor abciximab given before stenting in patients with AMI improved outcomes, resulting in better TIMI flow immediately following stenting and at six months, better recovery of left ventricular function at six months, and lower MACE at 30 days and six months. ${ }^{2}$

Stone and colleagues, in the recently completed CADILLAC trial, evaluated the combined role of stents and abciximab and documented the lowest event rates of any AMI trial to date ( 30 day mortality $2.0 \%$, reinfarcation $0.7 \%$, ischaemic TVR $3.3 \%$, and disabling stroke $0.2 \%){ }^{3}$ CADILLAC, like Stent PAMI, found that stents reduced six month TVR and MACE, but unlike Stent PAMI, found no degradation of TIMI flow and no worse (slightly better) survival in patients treated with new generation stents compared to balloon angioplasty alone. CADILLAC, like ADMIRAL, found that abciximab was associated with fewer ischaemic events and lower 30 day MACE, although the benefit was seen mostly in non-stented patients and the benefit was not as pronounced as in the ADMIRAL trial, which enrolled a higher risk patient population and gave abciximab before percutaneous coronary intervention (PCI).

The primary objective of reperfusion treatment for AMI for nearly two decades has been the achievement and maintenance of brisk coronary flow in infarct artery. Mechanical reperfusion combined with adjunctive treatment with stents and platelet glycoprotein IIb/IIIa inhibitors has achieved this objective, with TIMI 3 flow rates of $>90-95 \%$, reinfarction rates of $<1 \%$, and late infarct artery reocclusion rates of $<5 \%$. However, despite achieving very high rates of coronary reperfusion, recent data using sensitive markers of myocardial reperfusion, such as contrast echocardiography, myocardial blush assessment with angiography, and electrocardiographic ST segment resolution, indicate that about $50 \%$ of patients who achieve TIMI 3 coronary flow fail to achieve optimal myocardial reperfusion, and these patients have increased complications and worse outcomes. ${ }^{4}$ The reasons for suboptimal myocardial reperfusion are not fully understood, but likely include distal embolisation of plateletrich microthrombi as well as microcirculatory dysfunction.

\section{ADJUNCTIVE MEASURES}

Adjunctive measures to improve myocardial reperfusion have included both pharmacologic and mechanical strategies. Pharmacologic strategies have been directed primarily at platelet glycoprotein IIb/IIIa inhibition with the expectation that dissolution of the platelet-rich microthrombi in the diseased epicardial segment will reduce obstruction in the distal microvasculature. Neumann and colleagues evaluated patients undergoing stenting for AMI and demonstrated that both peak flow velocity in the infarct related artery and regional wall motion index in the infarct zone were significantly improved at two weeks in abciximab treated patients compared to controls. ${ }^{5}$ Similarly, the ADMIRAL and CADILLAC trials both showed clinical benefit with abciximab, although only the ADMIRAL trial showed improved left ventricular function at six months.

\footnotetext{
Abbreviations: ADMIRAL, abciximab before direct angioplasty and stenting in myocardial infarction regarding acute and long-term follow-up; $\mathrm{AMI}$, acute myocardial infarction; CADILLAC, controlled abciximab and device investigation to lower late angioplasty complications; FINESSE, facilitated intervention with enhanced reperfusion speed to stop events; GUSTO global use of strategies to open occluded coronary arteries; MACE, major adverse cardiac events; PAMI, primary angioplasty in myocardial infarction; $\mathrm{PCl}$, percutaneous coronary intervention; SAFER, saphenous vein graft angioplasty free of emboli randomized trial; SPEED, strategies for patency enhancement in the emergency department; TIMI, thrombolysis in myocardial infarction; TVR, target vessel revascularisation
} 
There are several mechanical strategies under evaluation which may enhance myocardial reperfusion. The X-sizer thrombectomy catheter (EndiCOR Medical, San Clemente, California, USA) is designed for selective removal of thrombus and atheromatous debris before stenting. Cox and colleagues recently reported results using the X-sizer catheter before stenting in patients with AMI and found improved tissue perfusion compared to historical controls as measured by angiographic myocardial blush scores. ${ }^{6}$ Randomised trials are planned in an attempt to validate these findings. The Guardwire System (Percusurge Inc, Sunnyvale, California, USA) employs a balloon inflated in the coronary artery distal to the target lesion before PCI to prevent distal embolisation. The atheromatous and thrombotic debris captured proximal to the balloon are removed with aspiration following stent deployment. The SAFER trial, presented by Baim and colleagues at the Transcatheter Cardiovascular Therapeutics Scientific Sessions in Washington DC last year, documented a significant reduction in embolisation and periprocedural infarction using the Guardwire with elective saphenous vein graft intervention. The potential for benefit in AMI is substantial, but placement of the Guardwire in an occluded artery with no distal visualisation remains problematic. The Filterwire (Boston Scientific/EPI, Santa Clara, California, USA) employs a filter mounted on a self expanding nitinol ring which is deployed in the coronary artery distal to the target lesion before PCI. Atheromatous and thrombotic debris are captured in the filter at the time of stent deployment, after which the filter is removed. This device has the potential to prevent distal embolisation and enhance tissue reperfusion, but is yet to be studied in AMI. Other novel devices employing thrombectomy or distal protection are in the planning stages of clinical trials.

\section{FACILITATED PCI}

A major limitation of mechanical reperfusion is lack of immediate availability. This lack of availability and the realisation that an optimum reperfusion strategy will need to incorporate mechanical reperfusion as part of that strategy has led to a great deal of interest in pharmacologic reperfusion combined with mechanical reperfusion or facilitated PCI. Facilitated PCI is the use of pharmacologic reperfusion to establish TIMI 3 flow as rapidly as possible followed by immediate PCI to maximise TIMI 3 flow and to stabilise the ruptured plaque. Previous studies had found that PCI following thrombolytic treatment was not beneficial and potentially harmful, but more recent experience using coronary stents and platelet glycoprotein IIb/IIIa inhibitors have found that PCI can be performed safely and effectively after pharmacologic reperfusion treatment. The SPEED trial found that PCI following thrombolytic treatment or half dose thrombolytic treatment combined with platelet glycoprotein IIb/IIIa inhibitors was associated with superior outcomes compared to pharmacologic reperfusion treatment alone. ${ }^{7}$ The PAMI investigators and the Moses Cone Hospital Registry documented that patients undergoing primary PCI for AMI who arrive at the catheterisation laboratory with an open versus a closed infarct artery have higher procedural success rates, smaller infarct size, better recovery of left ventricular function, and lower early and late mortality. ${ }^{89}$ These studies raise hope that facilitated PCI may improve outcomes over primary PCI alone, and this hypothesis will be tested in the FINESSE trial. This approach would be ideal for patients presenting at hospitals without interventional facilities to allow time for transfer for mechanical intervention.
Although there is much enthusiasm for facilitated PCI, a word of caution is needed. The major objective of facilitated PCI is to reduce the time delay inherent with mechanical reperfusion, but time to reperfusion appears to be less important with primary PCI than with thrombolytic treatment. ${ }^{10}$ Also, combination pharmacologic treatment using half dose thrombolytics and platelet glycoprotein IIb/IIIa inhibitors, which is being tested in facilitated PCI trials, has an increased bleeding risk and the same finite risk of intracranial haemorrhage as standard thrombolytic treatment as documented in the recent GUSTO V trial. ${ }^{11}$ Whether the benefit of facilitated PCI will be sufficient to outweigh these risks and to justify the increased costs is uncertain.

The past two decades have witnessed gradually improving outcomes with reperfusion treatment for AMI, beginning with first generation thrombolytics, followed by second and third generation thrombolytics combined with aspirin, heparin, and now platelet glycoprotein IIb/IIIa inhibitors, and most recently with mechanical reperfusion combined with stents and platelet glycoprotein IIb/IIIa inhibitors. New frontiers with reperfusion therapy will focus on improving reperfusion at the level of the microcirculation and combining pharmacologic with mechanical reperfusion to expand the benefits of mechanical reperfusion to a much broader patient population. This synergy between pharmacologic treatment and a variety of novel mechanical approaches holds promise to further improve outcomes with reperfusion treatment for AMI.

\section{Authors' affiliations}

B R Brodie, T D Stuckey, Greensboro, North Carolina, USA

\section{REFERENCES}

1 Grines CL, Cox DA, Stone GW, et al. Coronary angioplasty with or without stent implantation for acute myocardial infarction. N Engl J Med 1999;341:1949-56.

2 Montalescot G, Barragan T, Wittenberg $O$, et al for the ADMIRAL Investigators. Platelet glycoprotein Ilb/llla inhibition with coronary stenting for acute myocardial infarction. N Engl J Med 2001;344:1895-903

3 Stone GW, Grines CL, Cox DA, et al. A prospective, multicenter, international randomized trial comparing four perfusion strategies in acute myocardial infarction: Principal report of the controlled abciximab and device investigation to lower late angioplasty complications (CADILLAC) trial [abstract]. J Am Coll Cardiol 2001;37(No 2 suppl A):342A.

4 Van't Hof AWJ, Liem A, de Boer M, et al. Clinical value of 12-lead electrocardiogram after successful reperfusion therapy for acute myocardial infarction. Lancet 1997:350:615-19.

5 Neuman FJ, Blasini R, Schmitt C, et al. Effect of glycoprotein Ilb/Illa receptor blockade on recovery of coronary flow and left ventricular function after the placement of coronary artery stents in acute myocardial infarction. Circulation 1998;98:2695-701.

6 Cox D, Stuckey T, Low R, et al. Adjunctive thrombectomy combined with stenting for AMl: the EndiCOR X-sizer AMI registry [abstract]. J Am Coll Cardiol 2001;37(No2 Suppl A):306A.

7 Herrmann HC, Moliterno DJ, Ohman EM, et al. Facilitation of early percutaneous coronary intervention after reteplase with or without abciximab in acute myocardial infarction: results from the SPEED (GUSTO-4 Pilot) trial. J Am Coll Cardiol 2000;36:1989-96.

8 Stone GW, Cox D, Garcia E, et al. Normal flow (TIMI-3) prior to mechanical reperfusion therapy is an independent determinant of surviva in acute myocardial infarction - analysis from the PAMI trial. Circulation (in press)

9 Brodie BR, Stuckey TD, Hansen C, et al. Benefit of coronary reperfusion before intervention on outcomes after primary angioplasty for acute myocardial infarction. Am J Cardiol 2000;85:13-18.

10 Brodie BR, Stuckey TD, Wall TC, et al. Importance of time to reperfusion for 30-day and late survival and recovery of left ventricular function after primary angioplasty for acute myocardial infarction. J Am Coll Cardiol 1998:32:1312-19.

11 The GUSTO V Investigators. Reperfusion therapy for acute myocardial infarction with fibrinolytic therapy or combination reduced fibrinolytic therapy and platelet glycoprotein Ilb/Illa inhibition: the GUSTO V randomized trial. Lancet 2001;357:1905-14. 